\title{
Chemometrics and Hyperspectral Imaging Applied to Assessment of Chemical, Textural and Structural Characteristics of Meat
}

\author{
Marlon M. Reis ${ }^{1}$, Robbe Van Beers ${ }^{4}$, Mahmoud Al-Sarayreh ${ }^{3}$, Paul Shorten², Wei Qi Yan³, \\ Wouter Saeys ${ }^{4}$, Reinhard Klette ${ }^{3}$, and Cameron Craigie ${ }^{2}$ \\ ${ }^{1}$ AgResearch, Palmerston North, New Zealand \\ ${ }^{2}$ AgResearch, Hamilton, New Zealand \\ ${ }^{3}$ Auckland University of Technology, Auckland, New Zealand \\ ${ }^{4} \mathrm{KU}$ Leuven, Department of Biosystems, MeBioS, Leuven, Belgium
}

Abstract:

Spectroscopy in the visible near-infrared spectral (Vis-NIRS) range combined with imaging techniques (hyperspectral imaging, HSI) allows assessment of chemical composition, texture, and meat structure. The use of $\mathrm{HSI}$ in the meat and food industry has observed a significant growth in the last decade, yet its use for assessment of meat it is not optimal yet. The application of HSI for assessment of meat is reviewed with focus on its ability to capture meat unique chemical and structural characteristics. While HSI is widely used for assessment of chemical composition, a limited number of evidences on its ability to handle the effect of different sources of variation on the assessment is found. The use of spatially resolved spectroscopy has been able to detect structural information related to animal background, muscle type, rigor process and ageing. Similarly the use of texture features seem to capture unique characteristics of meat.

Keywords: Hyperspectral imaging; chemometrics; light scattering; meat; texture; spatially resolved spectroscopy 


\section{Introduction}

Factors including on farm practices, animal background and handling from farm to slaughter can all have significant impacts on meat product quality. Except perhaps in a well-established vertically integrated supply chain, meat processors have little influence over the animals they process. Despite target weight specifications and pricing incentives wide variation can be observed in carcass weight and fatness for example. Following slaughter, the rigor process commences leading to $\mathrm{pH}$ decline and along with several other biochemical changes including the rate and extension of $\mathrm{pH}$ decline, proteolysis and protein oxidation post slaughter that affect the release of water (Huff-Lonergan \& Lonergan, 2005). As the rigor progress, space for water to be held in the myofibrils is reduced and fluid can be forced into the extramyofibrillar zone (Huff-Lonergan \& Lonergan, 2005). At rigor, shortening of muscle fibres occurs negatively influencing meat quality (i.e., tenderness); however, proteolytic degradation of the meat proteins becomes favourable to the attributes such as meat tenderness (Tornberg et al., 2000). The relevant level of shortening and proteolytic breakdown of muscle proteins is dependent on chilling conditions and the level of stress ante-mortem as well as the course of $\mathrm{pH}$ decline prior to fully developed rigor (Tornberg et al., 2000). In summary, the rigor process involves a combination of biochemical and structural changes that are dependent on processing conditions (e.g., chilling rate, electrical stimulation) and animal background (e.g., glycogen reserve at slaughter, muscle fiber distribution and composition). Post rigor, a new series of biochemical and structural changes will take place in the meat due to ageing, storage temperature (e.g. freezing/thawing) and bacterial growth as well as the interactions among these factors. As a result of the rigor and ageing processes, meat owns unique chemical and structural characteristics that are uniquely and directly associated with its sources in the way it was processed, handled and transported to consumers. The detection of these unique chemical and structural characteristics offers an opportunity for the assessment of meat integrity at the stages of production, including assessment of quality, shelf-life and authenticity.

Spectroscopy in the visible and near infrared spectral (Vis-NIRS) range is based on the interaction of electromagnetic radiation with matter. When a beam of light is directed onto the meat, a part of it will have little interaction with the sample being reflected on its surface while the other part will travel through the meat, interacting with it, being scattered and absorbed (Dahm \& Dahm, 2001; Dahm \& Dahm, 2013; Jacques, 2013; Mollazade et al., 2012; Saeys et al., 2010). Four processes take place in this case (Mollazade et al., 2012), Figure 1: 1) Light absorption; 2) regular reflectance (specular) where the light incident angle with the meat surface is equal with the angle at which it is reflected, meaning little or no interaction with meat; 3) external diffuse reflectance, which capture information about the surface of the meat; and 4) light scattering, also due to interaction of light with the sample. The outgoing 
light from the sample surface results from scattering inside of the sample. The detection of the outgoing photons allows the assessment of how much was lost by absorbing/scattering as well as the amount reflected (specular and external diffuse). The ratio between the amount of outgoing photons from the sample to the amount of incident photons is commonly used as a measure of how much was lost by absorption and scattering as well as the amount reflected. Both absorption and scattering are wavelength dependent, making the use of the entire Vis-NIR spectrum a rich source of information about the chemical and structural characteristics of meat, which offers an opportunity for the assessment of meat integrity based on non-invasive approaches.

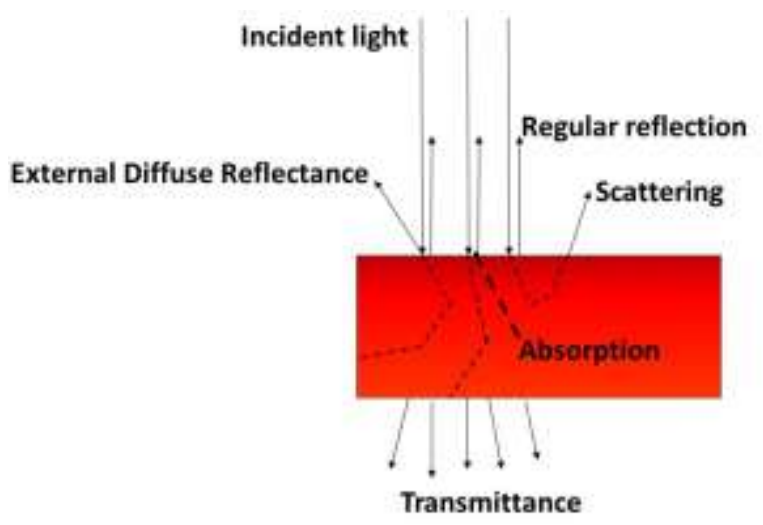

Figure 1. Distribution of incident light in a sample (Mollazade, et al., 2012).

HSI has been used in several applications for meat assessment showing results in the quality attributes as well as safety and authenticity (Elmasry et al., 2012; Feng et al., 2018; Kamruzzaman et al., 2013; Kamruzzaman et al., 2015; Liu et al., 2017; Xiong et al., 2014). HSI-based techniques have also been used to assess scattering and applied for predicting attributes which are associated with meat structure such as tenderness (Cluff et al., 2013; Peng et al., 2009; Ranasinghesagara et al., 2010; Wu et al., 2012; Wu et al., 2010). The use of hyperspectral imagining in the meat and food industry has observed a significant growth in the last decade as revealed in recent literature reviews. Cheng et al. (2017) reviewed application of HSI based on use of multivariate analysis for prediction of tenderness, colour attributes, drip loss, water-holding capacity, firmness, springiness, $\mathrm{pH}$ as well as meat classification regarding grading, muscle discrimination ad differentiation between fresh and frozen meat products(Cheng et al., 
2017). Similarly, Chen et al. (2017) described studies applying HSI combined with multivariate analysis for prediction of meat chemical composition. While Siche et al. (2017) reviewed HSI applied to assessment of quality attributes such as tenderness as well food and safety (Siche et al., 2016). These reviews describe well the application of the standard approach to HSI combined with classical multivariate analysis. However, less attention is given to the HSI underlying principles allowing development of new measuring techniques to detect aforementioned factors affecting the unique chemical and structural characteristics for assessment of meat integrity at the stages of production, including assessment of quality, shelf-life and authenticity. Thus in this paper the application of hyperspectral imaging for assessment of chemical, textural and structural characteristics of meat is reviewed with focus on its ability to capture unique chemical and structural characteristics of meat. Our motivation is to identify gaps and formulate future research needed to achieve optimal use of hyperspectral imaging in assessment of meat with the development of new experimental techniques and modelling approaches.

\section{Hyperspectral imaging for assessment of chemical, textural and structural characteristics of meat}

\subsection{Assessment of meat chemical composition and Chemometrics}

The most common approach used in HSI to assess chemical composition is based on reflectance measurements, where an exposed surface of the sample is illuminated and light emerging from the same surface is detected. The technique of choice is based on the ratio between the intensity of outgoing light and the intensity measured in a reference material considered as $100 \%$ of reflectance. This technique works under the assumption that the effect of specular reflectance, external diffuse reflectance and internal scattering (the part which is scattered internally and is not detected) in detected light intensity is similar among samples. In the cases, where this assumption does not fully apply, pre-processing of the spectra is performed to reduce the impact of these effects (Cheng et al., 2017). Then, a Chemometric model is fitted to relate the detected spectra with the attributes of interest (Cheng et al., 2017).

The most common methods for analysis of hyperspectral data in meat include (Cheng et al., 2017): Principal Component Analysis (PCA); Partial Least Squares Regression (PLSR); Multi Linear Regression (MLR); Support Vector Machine (SVM); and Artificial Neural Network (ANN). These methods are adaptable either for the development of predictive or classification models (Amigo et al., 2015; Geladi, 2003; Juan et al., 2004). PCA is commonly used for exploratory analysis. Predictive models are used to estimate a value for an attribute(s) of interest, e.g. fat content, from the hyperspectral data. Classification models aim to assign a category, e.g. fresh, to sample using the hyperspectral data. While 
exploratory analysis is perform to identify patterns in the data. Prior the application of these methods the hyperspectral data undergoes several steps of processing in spectral and spatial domain (Amigo et al., 2015; Rinnan et al., 2009b). These pre-processing methods are dedicated to deal with effect of variability in the sample that affects the NIR spectra but are not easily modelled. The pre-processing methods mostly used for the spectral domain include: Smoothing and derivatives(Savitzky \& Golay, 1964), Standard Normal Variate (SNV)(Barnes et al., 1989; Feng \& Sun, 2013), Multiplicative Scatter Correction (MSC) (Rinnan et al., 2009a), Orthogonal Signal Correction (OSC)(Sjöblom et al., 1998). Smoothing the spectral data is important step to remove the noise. In general, the derivatives $\left(1^{\text {st }}\right.$ and $2^{\text {nd }}$ ) are used to emphasize the spectral information. The first derivative is used to remove the additive baseline in signal and the second derivative is utilized to remove the linear baseline (multiplicative) from the signal(Martens \& Naes, 1992). Reduction of the effect of regular reflectance and external diffuse reflectance on the spectral data is commonly performed with MSC, SNV, baseline correction, and Detrending (Keresztes et al., 2016). Methods applied to spatial domain ( $\mathrm{x}$ and $\mathrm{y}$ coordinates) includes,: Homogeneous smoothing (averaging); Gaussian smoothing; Median smoothing; Bilateral smoothing, and wiener filter, (Klette, 2014). These methods considers the local neighbors (4, 8, etc.) of each pixel at each band of the hyperspectral data.

Table 1 presents the application of chemometric methods for the prediction of intramuscular fat content (IMF\%) using HSI with samples $(n=2454)$ of lamb loin $(m$. longissimus lumborum) collected in three consecutive years at different meat processing plants. Details of experimental methods used for data collection has been discussed elsewhere (Craigie et al., 2017). Ten different methods were evaluated including: Partial least squares regression (PLSR) with latent variables selection based on the adjusted wold's R criterion with thresholds on unity (AW) and 0.99 (AW0.99)(Li et al., 2002), Gaussian process regression (GPR)(Chen, Morris, et al., 2007; Gibson et al., 2012; Verrelst et al., 2013), Support Vector Machine (SVM)(Borin et al., 2006; Chen, Zhao, et al., 2007; Zhang et al., 2008), PLSR with wavelength selection according to competitive reweighted adaptive sampling (CARS)(Li et al., 2009) and variable importance in projection (VIP)(Chong \& Jun, 2005), Multiple Linear Regression (MLR), Stepwise Multiple Linear Regression (SMLR)(Hastie et al., 2008), lasso regularization for linear regression (LASSO)(Hastie et al., 2008) and Robust Multiple Linear Regression (RMLR)(Hastie et al., 2008). SNV was used as pre-processing method. Overall similar performance was obtained independently of the method used.

Table 1 The performance of different calibration models. The calibration is based on $66 \%$ data with validation carried out on the remaining of the data. PLSR with latent variable selection is based on the Adjusted Wold's R criterion with thresholds on unity \& 0.99, GPR, SVM, PLSR with wavelength selection 

from $0.86 \%$ to $9.48 \%$, with standard deviation of 0.82 and mean $2.67 \%$.

\begin{tabular}{|l|l|l|l|l|}
\hline HSI, IMF\% & $\begin{array}{l}\mathrm{R}^{2} \text { calibration } \\
(\mathrm{N}=1628)\end{array}$ & RMSE calibration & $\begin{array}{l}\mathrm{R}^{2} \text { validation } \\
(\mathrm{N}=826)\end{array}$ & RMSE validation \\
\hline PLSR (AW) & 0.74 & 0.41 & 0.71 & 0.46 \\
\hline PLSR (AW0.99) & 0.71 & 0.44 & 0.70 & 0.46 \\
\hline GPR & 0.79 & 0.37 & 0.72 & 0.45 \\
\hline SVM & 0.74 & 0.41 & 0.68 & 0.47 \\
\hline PLSR (CARS) & 0.74 & 0.41 & 0.70 & 0.46 \\
\hline PLSR (VIP) & 0.66 & 0.48 & 0.67 & 0.48 \\
\hline MLR & 0.77 & 0.42 & 0.70 & 0.46 \\
\hline SMLR & 0.73 & 0.43 & 0.71 & 0.45 \\
\hline LASSO & 0.72 & 0.43 & 0.71 & 0.45 \\
\hline RMLR & 0.75 & 0.41 & 0.69 & 0.47 \\
\hline
\end{tabular}

Chen et al. (2016) recently reviewed the use of hyperspectral imaging for assessment of chemical information in meat(Chen, Sun, et al., 2016). Moisture, fat content and composition, protein and pigments are the most common attributes evaluated, where the performance reported on the basis of $R^{2}$ is in general above 0.8 (Chen, Sun, et al., 2016). While these feasibility studies have been showing the ability of HSI to predict chemical composition of meat (Chen, Sun, et al., 2016) there is a lack of studies demonstrating the robustness of the system for use at meat processing plants. The performance based on $\mathrm{R}^{2}$ for prediction of fat content in beef and lamb, reported in other studies, varying between 0.84 and 0.93 (Chen, Sun, et al., 2016) was higher than observed in our study (Table 1). Kamruzzaman et al. (2012) used HSI to predict fat content in lamb using 126 samples (42 animals $\times 3$ muscles, semimembranosis, semitendinosus and longissimus dorsi) and obtained a very good performance $\left(R^{2} \mathrm{cv}\right.$ $=0.91, R^{2} p=0.88$ and SEP $=0.40 \%$ )(Kamruzzaman et al., 2012). Pu et al. (2014) evaluated the same data set as Kamruzzaman et al. (2012), but with alternative modelling approach and observed and a slight increase on prediction performance $\left(R^{2} c v=0.95, R^{2} p=0.98\right)(P u$, Sun, Ma, Liu, \& Kamruzzaman, 2014). However, the SEP in our study ( $n=2454$ samples) is relatively similar to that obtained by Kamruzzaman et al. (2012) ( $n=126$ samples). The study by Kamruzzaman et al. (2012) was performed with similar range of fat concentration ( 0.74 to $7.62 \%$, mean: $2.42 \%$ and sandard devition 1.33 ) as reported in Table 1 , and the difference in performance between studies might have results from effects of other sources of variation (e.g different processing plants, seasonality) not included in the study of Kamruzzaman et al. (2012). 
When different sources of variation are present (e.g. different processing plants, seasons, animal background) for large number of samples the prediction performance is reduced. There is a knowledgegap on understanding how the effect of these sources of variation impact the performance of HSI for prediction of chemical composition and approaches are needed to improve performance. As discussed in the section 2.2 and 2.3 meat has a defined structure affecting the spatial domain of the data which has also effect on spectral domain. But, the majority of approaches described for development of model for HSI either as predictive or for classification of meat are based on methodologies developed for NIRS and that do not take into consideration the interactions between spectral and spatial domain.

Recently, deep learning models have been introduced to HSI field and other fields like image, languages, and speech processing(Lecun et al., 2015; Schmidhuber, 2015). Deep learning model 'learn' deep features in a hierarchical way and produce features have with a high level of abstraction, complexity, and invariance to the local change in the input data(Bengio et al., 2013). In general, there are many deep neural network architectures proposed in the field of hyperspectral imaging such as deep belief networks (DBNs), deep Boltzmann machines (DBMs), stacked autoencoder (SAEs), and convolutional neural networks (CNNs). A deep learning approach to capture spectral and spatial features was proposed for the first time in hyperspectral imaging by (Chen et al., 2014) and has been attracting attention in the last few years(Li et al., 2017; Makantasis et al., 2015). SAE network is used to extract deep features followed by logistic regression for doing the classification part, where the considered features were spectral (1D vector), spatial (flatted nxn regions in PCA space), and joint spectral-spatial by stacking the feature vectors as a one feature vector (Chen et al., 2014). Similar methodology was implemented for extracting the features using DBN with using of restricted Boltzmann machine for building the network and estimating the weights and parameters(Li et al., 2017). In fact, these methods (SAE, and DBN) achieve a high performance compared with a shallow machine learning methods like SVM, KNN, ANN, etc., but still don't consider the spatial information in 2D structure, since converting the $n x n$ regions into $1 D$ vector destroy the meaning of spatial information. $C N N$ comes to solve the problem of spatial information, where it consider the sample as 2D coordinates. The CNN implementation for classifying hyperspectral images using the only spectral information, a 1D vector (spectrum) as input layer similar, was one of the first application of CNN (Hu et al., 2015). CNN implemented for investigating the different types of features (spectral, spatial, and joint of them) using a 3D CNN has been reported (Chen, Jiang, et al., 2016). The motivation of using deep learning in hyperspectral imaging analysis applied to meat is that deep learning allows modelling of several levels of interaction such as spectral-spatial, variation in light scattering, spectral variation and sample presentation such as sample moving and rotation. Moreover, it can handle the raw input data as input and extract the features by itself, where a pre-processing and features extraction are not needed. 
207 Therefore new modelling approaches based 3D CNN is expected allow to maximize the capabilities of $208 \mathrm{HSI}$ on assessment of meat. Recently, the use of 3D-CNN combined with spectral features has been 209 described showing the possibility to model hyperspectral data from meat in combination with several 210 streams of information allowing complex interaction within the dataset to be modelled(Al-Sarayreh et 211 al., 2018). Further research should be dedicated to investigate how this type of approach would allow to combine spectral information, texture and scattering based measurement into a single model.

\subsection{Assessment of meat structural characteristics}

215 The structure of the muscle (converted to meat) presents several levels of complexity (Figure 2), that dependent on animal background and growth and that will be significantly affected by the process of conversion of the muscle into meat and ageing.

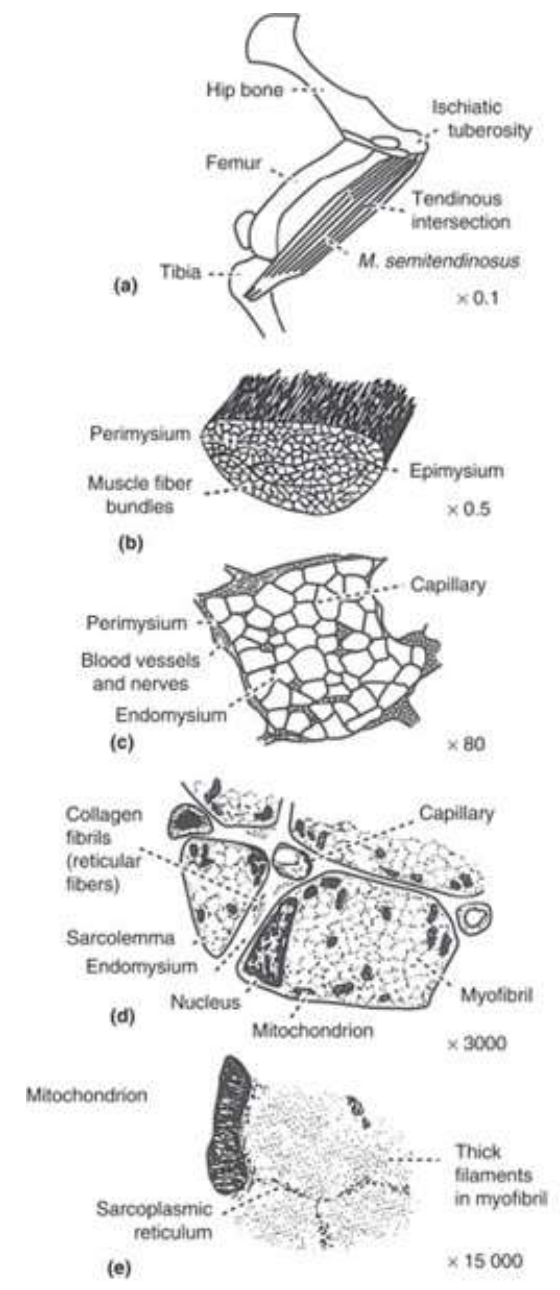


Figure 2 - Figure 2. Gross and ultrastructural anatomy of the sheep semitendinosus muscle (Astruc, 2014). The semitendinosus muscle lies caudally in the thigh region (a). Seen grossly in transverse section, perimysial strands course through the muscle and enclose muscle fiber bundles (b). Within a bundle, an endomysial connective tissue network is revealed by light microscopy to surround individual muscle fibers (c). Electron microscopic examination shows a network of sarcoplasmic reticulum enclosing myofibrils within the fibers (d). The two complete fibers drawn sectioned here are smaller than actual fibers in the sheep semitendinosus muscle. At a still higher magnification, a pattern of thick filaments is visible within each myofibril (e). Reprinted with permission of Elsevier, 2018.

As light travels through these different structures of the meat (Figure 2) it goes from areas of given refractive index (e.g. mitochondria) to another area of a different refractive index (e.g. collagen fibrils, Figure 2d), as result it changes its path and refraction of light takes place and leading to optical scattering (Mollazade et al., 2012). Optical scattering can be described either as scattering by particles that have a refractive index different from the surrounding medium, or as scattering by a medium with continuous but fluctuating refractive index(Jacques, 2013). The heterogeneous structure of tissue (Figure 2), that makes it a strong scatter, results in a complex light propagation problem very difficult or practically impossible to be solved theoretically(Mollazade et al., 2012). Thus behaviour of light propagation in a biological tissue, has been approached based on diffusion theory model and Monte Carlo simulation(Mollazade et al., 2012).

One approach to estimate the optical properties of the meat is based on spatially resolved spectroscopy

240 (Aernouts et al., 2011), where a beam of light is focused on a spot; and outgoing light on the 241 surroundings of this spot is scanned using HSI, Figure 3 (Mollazade et al., 2012). The variation on detected light intensity as the distance to the illumination spot is used to assess absorption/scattering in the sample (Aernouts et al., 2011). These approaches have been used for the assessment of structural characteristics of meat and quality attributes such as tenderness (Cluff et al., 2008; Cluff et al., 2013; Ranasinghesagara et al., 2010; Ranasinghesagara \& Yao, 2007; Xia et al., 2007).

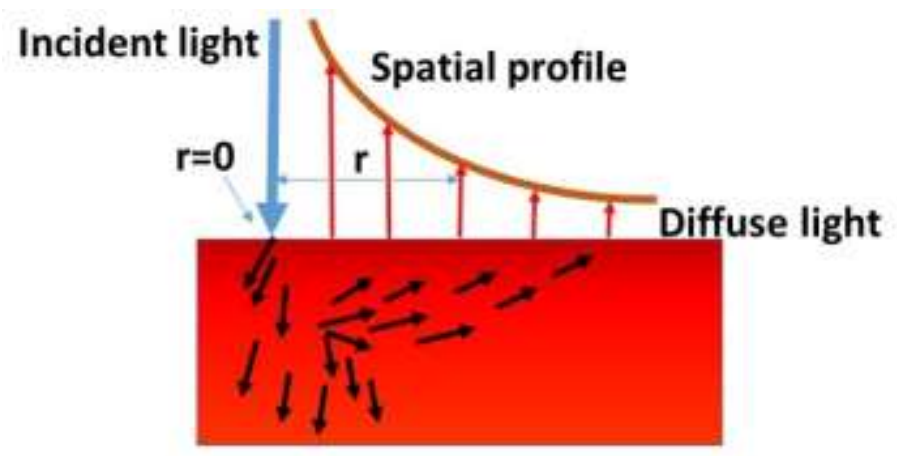


Figure 3 Figure 3. Measurement principle of the spatially resolved steady-state method for measuring the optical properties of the sample (Mollazade et al., 2012).

249

250

251

252

253

254

255

256

257

258

259

260

261

262

263

264

265

The optical properties of a tissue can be described in terms of the absorption and scattering coefficients. Absorption coefficient is a measure of the rate of decrease in the light intensity as it passes through a given substance(Mollazade et al., 2012). While scattering coefficient is the fraction of light scattered per unit distance in a participating medium(Mollazade et al., 2012). Anisotropy of scatter, characterizes tissue scattering in terms of the relative forward versus backward travel in thicker tissues where multiple scattering occurs in the direction of scatter. The anisotropy factor $(\mathrm{g})$ is calculated as function of angle existing between the direction of the photon before a scattering event and the direction after the scattering event (in the interval $[0, \pi]$ )(Mollazade et al., 2012). During the diffusion process, photons have a random movement through the medium in a sequence of strides of lengths and directions (Mollazade et al., 2012). Every stride being with a scattering event is equally likely to be taken in any direction(Mollazade et al., 2012). Reduced scattering coefficient is a description of the combined effect of scattering coefficient and average scattering angle as follows

Reduced scattering coefficient= (1-g) x Scattering coefficient, (Mollazade et al., 2012).

Spatially resolved spectroscopy has been used to estimate absorption and reduced scattering coefficient spectra of different beef samples based on light diffusion model(Mollazade et al., 2012; Van Beers et al., 2018), and other approaches to estimate these coefficients are reported by (Jacques, 2013).

Figure 4 presents the results of spatially resolved spectroscopy applied to two muscle types originated from bull and steers, where setup for data acquisition is described in (Van Beers, Aernouts, Reis, et al., 2017). A logarithmic (base 10) function was fitted to the reflectance as a function of the distance from the illumination spot. From this fitted function, two parameters characterizing the profiles were tuned: slope and intercept. These parameters were estimated for each wavelength (550 nm to $1700 \mathrm{~nm}$ ) resulting in profiles of slope and intercept per sample as shown in Figure 4. Reduced scattering coefficient shows an exponential decay with increase in the wavelength while absorption is characterized by peaks around specific wavelengths, see Figure 1 for example in (Van Beers et al., 2018). Thus the decrease in slope values and increase of intercept values as function of wavelength could be indication of changes in the scattering properties of the meat while the peaks are associated to changes in absorption. Steers show, in general, higher values of slope and lower values for intercept in Figure 4, and therefore lower relative reflectance and slower decay compared to bulls. Due to the overall higher bulk scattering, more photons exited close to the point of illumination as they cannot travel as deeper inside the tissue before being scattered and absorbed. This effect seems to be more evident in bulls. 
Similarly, the difference between muscles is observed for both steers and bulls, but is more significant for bulls. Samples from brachialis in bulls also show differences in the slope profiles, especially in the peak around $1100 \mathrm{~nm}$, which is much narrower than the others as well as the presence of a peak around $900 \mathrm{~nm}$. For intercepts, these peaks are observed at 800 and $1000 \mathrm{~nm}$. These spectral regions are related to overtones of $\mathrm{CH}$ molecular bond, but it is not clear how they are related to separation between muscles types for bulls. Overall, this application illustrates the ability to capture information about animal background and muscle type.
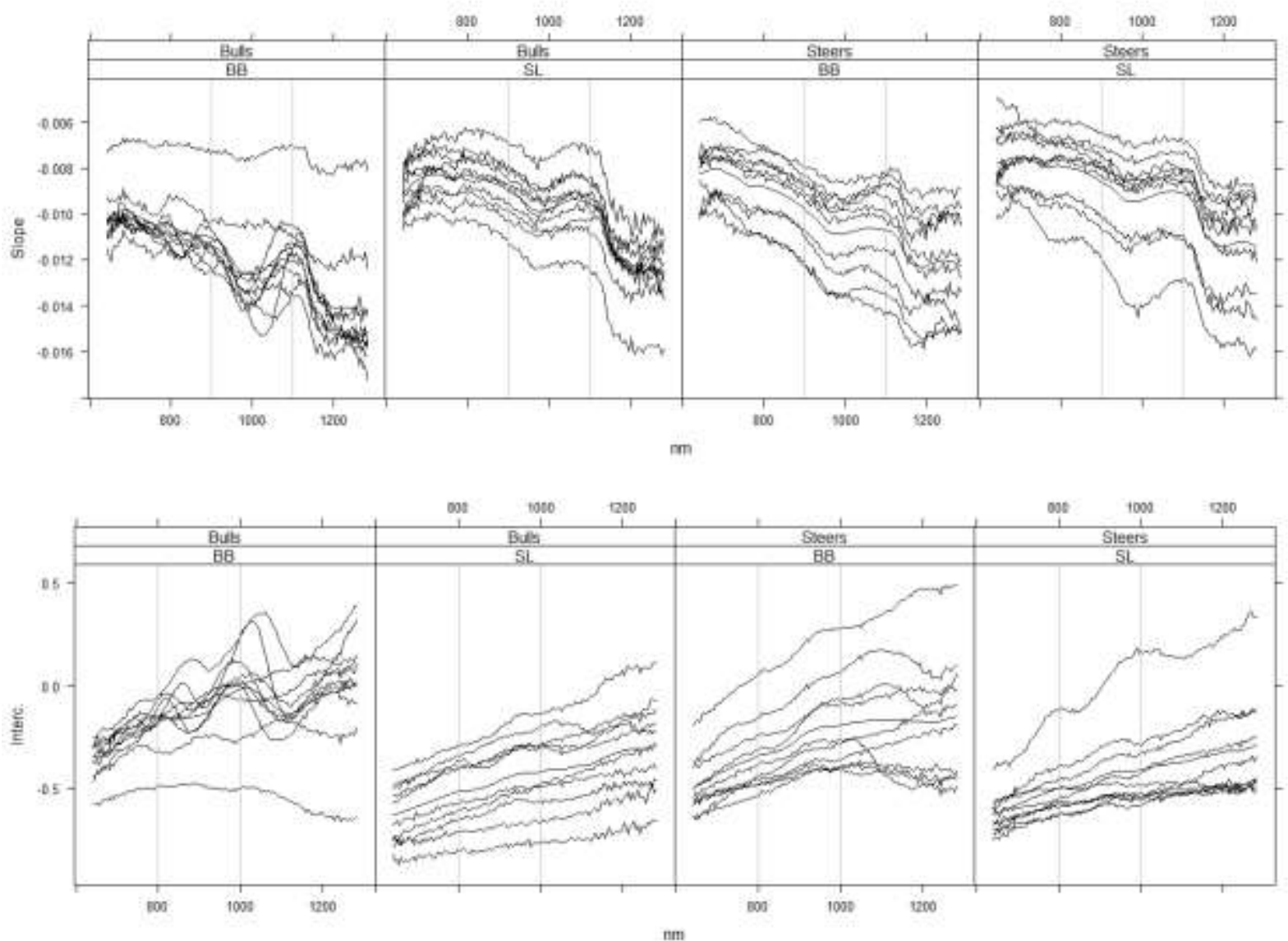

Figure 4. A logarithmic (base 10) function was fitted to the reflectance as a function of the distance from the illumination spot, see Figure 3 for illustration. From this fitted function, slope and intercept values were estimated for each wavelength. Profiles of slope (top panel) and intercept (bottom panel) as function of wavelength per animal category and muscle type ('BB' - Brachialis and 'SL' M. Soleus) are presented. The vertical lines in the top panel correspond to 900 and $1100 \mathrm{~nm}$ and 800 and $1000 \mathrm{~nm}$ at the bottom panel. 
reflection measurements acquired from muscle samples (Van Beers, Aernouts, Reis, et al., 2017). They suggested that this effect should result from the scattering of photons on aligned cylindrical microstructures (muscle fibers)(Van Beers, Aernouts, Reis, et al., 2017). Muscle fibers have large regular structures (e.g., mitochondria (300-900 nm), $t$-tubules, sarcoplasmic reticulum, A-band (region of overlap with both thin and thick myofilaments), I-band (region of thin myofilaments)) that repeats both radially and longitudinally along the fibre with a characteristic length of $\sim 500-1500 \mathrm{~nm}$ that are dependent on fiber type (Figure 8 from (Shorten \& Sneyd, 2009); Figure 17 from (Ogata \& Yamasaki, 1997)). The two muscles in Figure 4, brachialis and soleus, have very distinct distributions of fiber types with soleus having more than $90 \%$ of Type I fiber; meanwhile, brachialis has around $60 \%$ of Type I fiber. Thus, it is thicker than that of soleus. Differences in scattering properties among muscles types involving more similar distributions of fiber types have been reported (Van Beers et al., 2018; Xia et al., 2007). Hence, it can be proposed that fiber type distribution play a key role on the light scattering of the muscle, alongside other factors such as collagen fibers and myofibrils.

Xia et al. (2006) proposed that optical scattering properties in the muscle are dependent on the collagen content of the muscle since the sizes of collagen fibers and myofibrils in meat are all closer to or larger than the used optical wavelengths (visible and short-wave near infrared light). Xia et al. have also shown that optical scattering properties measured in whole muscle are related to changes in sarcomere structure (Xia et al., 2006). Xia et al. (2006) applied spatially resolved optical reflectance on whole muscle and observed that reduced scattering coefficients of post rigor psoas major samples increased with sarcomere length. Their results also suggest that the optical scattering measurements can characterize structure changes during rigor mortis development, where scattering coefficients decreased with time and the decay rate became slower after a certain period. It was proposed that permanent formation of cross-bridges is the dominant effect in altering scattering coefficients(Xia et al., 2006).

Overall these independent studies shows that the use of spatially resolved experiments base on hyperspectral imaging does provide a direct link to structural components of meat and is able to capture structural changes taking place from pre-rigor to post ageing. While most of studies described in the literature are based on laboratory type instruments, the introduction of a portable device has been described(Van Beers, Aernouts, Deserranno, et al., 2017) showing feasibility of using spatially resolved measurements at meat processing plants. Furthermore new hyperspectral devices, such as snapshot cameras(Geelen et al., 2013) that are portable, don't require sample translation and will allow the development of new portable devices for spatially resolved spectroscopy to be used at processing plants. Thus further investigation should be dedicated on developing measuring systems able to assess light scattering with other features capture by hyperspectral imaging. 


\subsection{Assessment of meat textural characteristics}

The texture of meat is formed by repeating units at different macro and microscopic scales, as illustrated in Figure 2 (Astruc, 2014), which are dependent on muscle type and species being affected by processing of the meat. Thus, the texture brings important information about the meat. Texture within the context of this review is defined as non-random arrangement of entities with given distribution of intensities and shapes, see Figure 2b,c for example, (Di Cataldo \& Ficarra, 2017).

A common model for extracting texture features in image is based on spatial relationships of adjacent pixels by calculating how often a pair of pixels with the same intensity values occur in an image (Klette, 2014). This is estimated by using the gray-level co-occurrence matrix (GLCM)(Klette, 2014). Statistical texture features can be extracted from GLCM matrix including (Haralick, 1979): homogeneity, contrast, inverse difference moment, entropy, energy and correlation. Contrast provides a measure of intensity or gray level variations between the reference pixel and its neighbour, where large contrast reflects large intensity differences in GLCM(Klette, 2014; Zayed \& Elnemr, 2015). Homogeneity measures how close the distribution of elements in the GLCM is to the diagonal of GLCM. As homogeneity increases, the contrast, typically, decreases(Klette, 2014; Zayed \& Elnemr, 2015). Entropy is the randomness or the degree of disorder present in the image. The value of entropy is the largest when all elements of the co-occurrence matrix are the same and small when elements are unequal(Klette, 2014; Zayed \& Elnemr, 2015). Energy is derived from the Angular Second Moment (ASM). The ASM measures the local uniformity of the gray levels(Klette, 2014; Zayed \& Elnemr, 2015). When pixels are very similar, the ASM value will be large. The Correlation feature characterizes the linear dependency of gray level values in the co-occurrence matrix(Klette, 2014; Zayed \& Elnemr, 2015).

Transform-based texture analysis (Di Cataldo \& Ficarra, 2017) has also being used in hyperspectral imaging. This type of approach transforms the image into a different space aimed to enhance texture properties and maximize the geometrical separability of different types of textures(Di Cataldo \& Ficarra, 2017). Among these approaches there are: Spatial domain filters, based on edge detection operators allowing to distinguish coarse from fine patterns; frequency domain filters, used to extract spatialfrequency components of the images detecting global texture properties such as coarseness, graininess, or repeating patterns; Gabor and wavelet transforms, are based on a similar principle to frequency domain filters but, wavelets uses different types of basis functions than the sinusoidal basis and is scale dependent; the Gabor transform is characterized by a Gaussian-shaped window function, which makes it better suited to represent spotted and concentric textures(Di Cataldo \& Ficarra, 2017). 
GLCM is typically applied to a single channel of an image and its use in hyperspectral data involves the estimation of features for each image in the hyperspectral cube or for selected images. It is also possible to use data-reduction methods such as PCA to reduce the spectral dimension and concentrate the information within few images. For example, Naganathan et al. (2015) investigated the use of hyperspectral image features for classification of beef samples according tenderness(Naganathan et al., 2015). They used descriptive statistical features including: Wavelet features; GLCM; Gabor features, Laws' texture features, and local binary pattern features. The features were extracted after reducing the dimension of hyperspectral images using PCA. The features extracted from the 2-day images were used to develop tenderness classification models for forecasting the 14-day beef tenderness. GLCM outperformed the other models and achieved a tenderness certification accuracy of $87.6 \%$, overall accuracy of $59.2 \%$, and an accuracy index of $62.9 \%$.

The combination of spectral and texture features, has been reported in applications related to authenticity, such as differentiation between free-range and broiler chicken meat (Xiong et al., 2015) and the differentiation between fresh and frozen-thawed pork meat (Ma et al., 2015; Pu et al., 2015; Pu, Sun, Ma, Liu, \& Cheng, 2014). Cheng et al. used GLCM features (contrast, correlation, energy, and homogeneity) in combination with spectral information for the assessment of freshness based on $\mathrm{K}$ values (Cheng et al., 2016). The performance $\left(R^{2}\right.$, validation) of predictions models were improved from 0.87 to 0.92 and shown an increase of at least $17.5 \%$ in predictive performance, when compared to models-based either on spectral or textural data alone(Cheng et al., 2016). Garrido-Novell et al. applied textural information from hyperspectral data to discriminate between pork, poultry and fish in processed animal protein meat. Garrido-Novell et al. (2018) expressed texture based on contrast, homogeneity, energy and correlation estimated from GLCM, but applied to all 212 wavelengths scanned in the hyperspectral image (Garrido-Novell et al., 2018). The integration of spectral and textural information into a single model also resulted in a higher rate of correct classification compared to the spectral model (92\% versus 83\%) (Garrido-Novell et al., 2018).

Recently Guo et al. (2018) proposed the use of bi-dimensional PCA for extraction of structural information and multi-features from hyperspectral data (Guo et al., 2018). Guo et al. observed that entropy values decrease with the increasing time of pork meat storage, where higher value of entropy indicates a more uniform meat surface (Guo et al., 2018). It was proposed that changes in the surfaces indicated by changes in entropy could result from protein degradation that causes damage in the integrity of the structure of muscle cells (Guo et al., 2018). In this case, the texture features of the hyperspectral image based on Gabor filters showed variations due to the storage time mainly in the visible spectral range associated with myoglobin (Guo et al., 2018). 
The texture of meat is formed by features at several spatial scales. Texture features in two different spatial scales can also be extracted with combined techniques, for example, combing superpixel segmentation (Achanta et al., 2012) with GLCM. The pixels in each superpixel share "similar" local spectral features, reflecting local spatial features (e.g., similar texture) in the image. The simple linear iterative clustering (SLIC)-superpixel (Achanta et al., 2012) segments are calculated by setting the segment region into given number of pixels ' $n$ ', which means that the size of each superpixel is around n pixels. SLIC-superpixel was originally proposed for colour images; with the similarity (using the Euclidean distance) among pixels assessed in RGB or CIE-LAB space, combined with the closeness of spatial coordinates. SLIC can be applied in the PCA space to capture spatial and spectral similarities combined. The scores from the first and most relevant principal components are used to produce images with channels as the input for SLIC. Euclidean distance is used as the similarity measure in the PCA space. Once superpixels are calculated, spectral and texture features estimated using GLCM are extracted for each superpixel(Al-Sarayreh et al., 2017). The spectral features correspond to spectra of representative pixels selected using Kennard-Stone algorithm applied to each superpixel(Al-Sarayreh et al., 2017). Figure 5 illustrates the methodology for extracting the texture features from those HSI images.
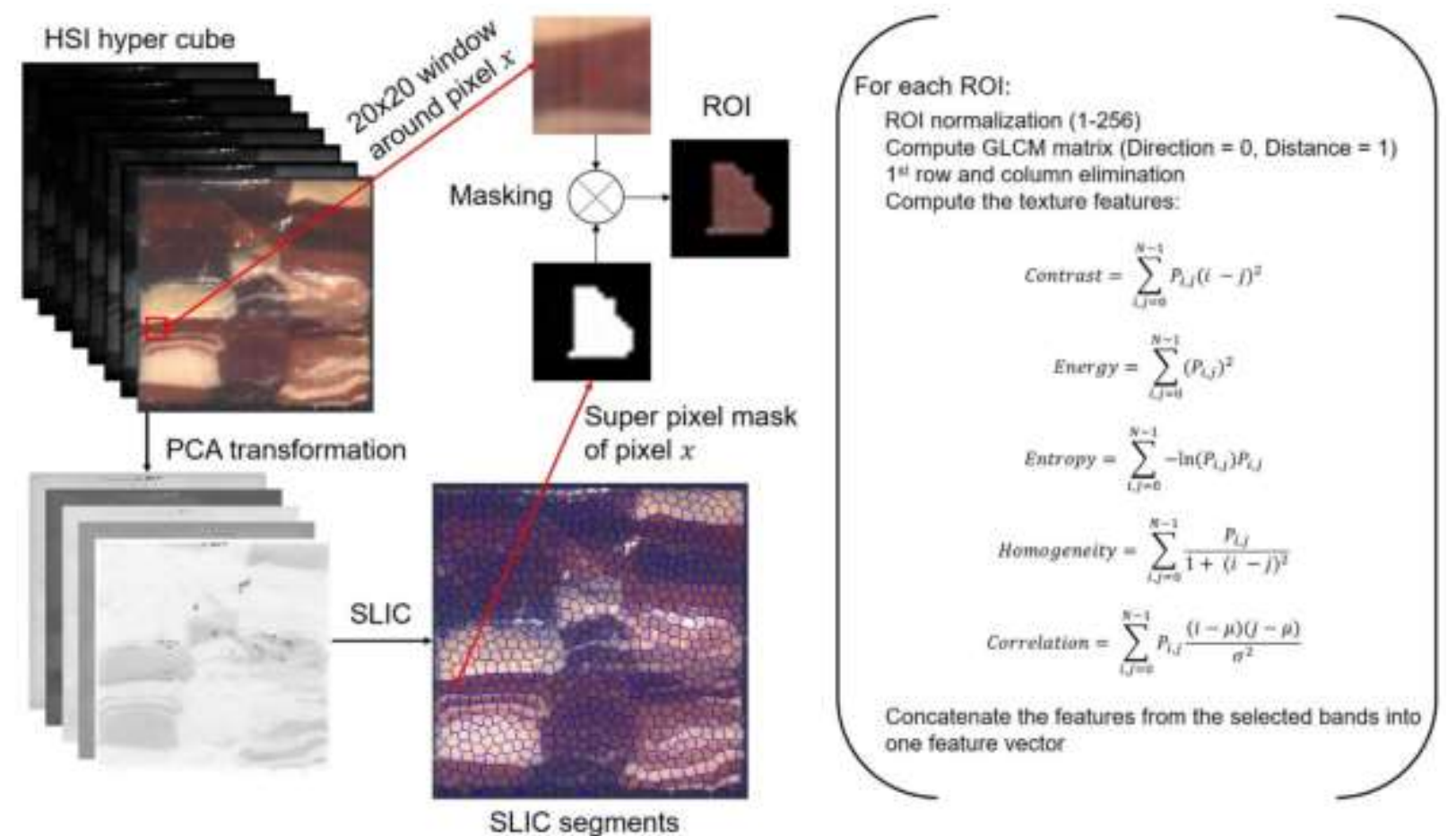

Figure 5. A representation of texture feature extraction from an $\mathrm{HSI}$ image, where $\mathrm{P}_{\mathrm{i}, \mathrm{j}}$ is an element $i, j$ of the normalized symmetrical GLCM, $\mathrm{N}$ is Number of gray levels (256), $\mu$ is the GLCM mean, and $\sigma$ is the standard deviation of the intensities of all reference pixels in the relationships 
423 Recursive feature elimination (RFE) (Ambroise \& McLachlan, 2002; Svetnik et al., 2004), for example, 424 allows the selection of a set of most significant image for making a distinction between classes (Al425 Sarayreh et al., 2017). In the RFE procedure, a random forest (RF) algorithm is used to estimate the importance of each image from data cubes in the classification task. The images found with the highest importance (rank) resulting from the RF model are selected. After that, texture features are computed for each superpixel at selected images by using the mentioned methodology above (Al-Sarayreh et al., 2017). Then, these sets of features are added to the selected spectral features of pixels inside the considered super-pixel, which significantly increases the performance of classifications models for species classification (Al-Sarayreh et al., 2017). In this case, it was possible to achieve an increase in precision from $71 \%$ to $82 \%$ on classification of lamb meat against others, and an increase from $87 \%$ to $92 \%$ on overall accuracy (Al-Sarayreh et al., 2017). The performance of the final model was found to be independent of status of the scanned samples (fresh unpacked, fresh packed, frozen unpacked, frozen packed, and frozen-thawed unpacked) (Al-Sarayreh et al., 2017).

These studies show the importance of using texture features calculated from hyperspectral images. However, the integration between spectral and texture features in modelling is still performed independently, i.e., the dependence between texture and spectral features is not expressed explicitly in a single model. The introduction of CNN, as reviewed in section 2.1, for image analysis creates an opportunity for this type of modelling. In this type of network, the data cube (height $\mathrm{x}$ width give the image dimensions and depth the number of channels, Figure 6-1-a) is first presented to the convolution step, where the filter with 3D size (covering all the channels, Figure 6-1-a) is passed over the image area to generate a feature map (Figure 6-1-b)(Fei-Fei et al., 2017; Lecun et al., 2015). The appropriate selection of this filter allows one to apply a function of channels (wavelengths in the hyperspectral data) and spatial distribution on the image space to estimate the interaction between effect of texture and spectral capture in the feature map. The full architecture of networks allows the connection of the features through pooling and connection among layers throughout the network. As results, it represents all levels of complexity of the hyperspectral data within a single model. For example, Gatys et al. introduced the concept of a nonlinear filter bank using convolutional neural networks to describe texture in images (Gatys et al., 2015; Wallis et al., 2017). Figure 6-4 presents an image resulting model developed by Gatys et al. applied to image in Figure 6-2, and the Figure 6-3 shows one of the features maps extracted from this model to illustrate the ability to capture features of HSI data. 
(1)

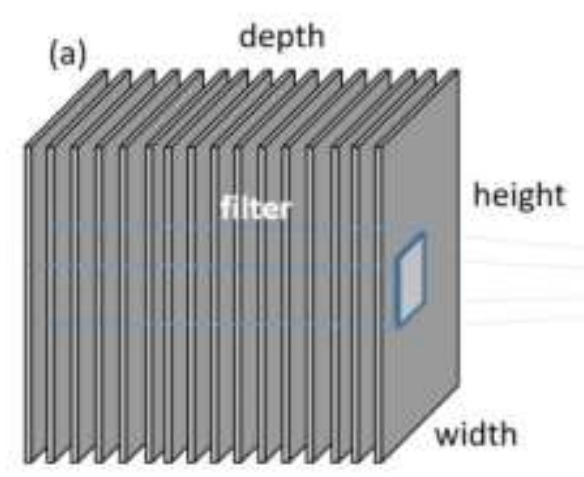

(3)

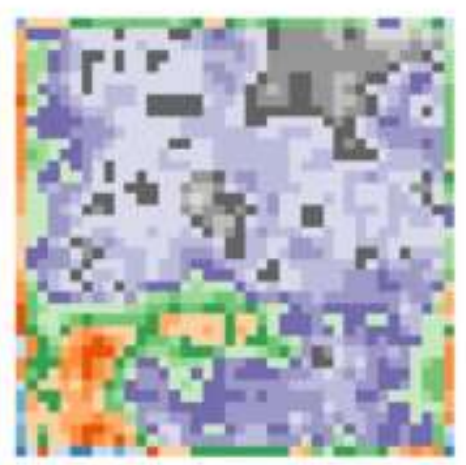

(2)
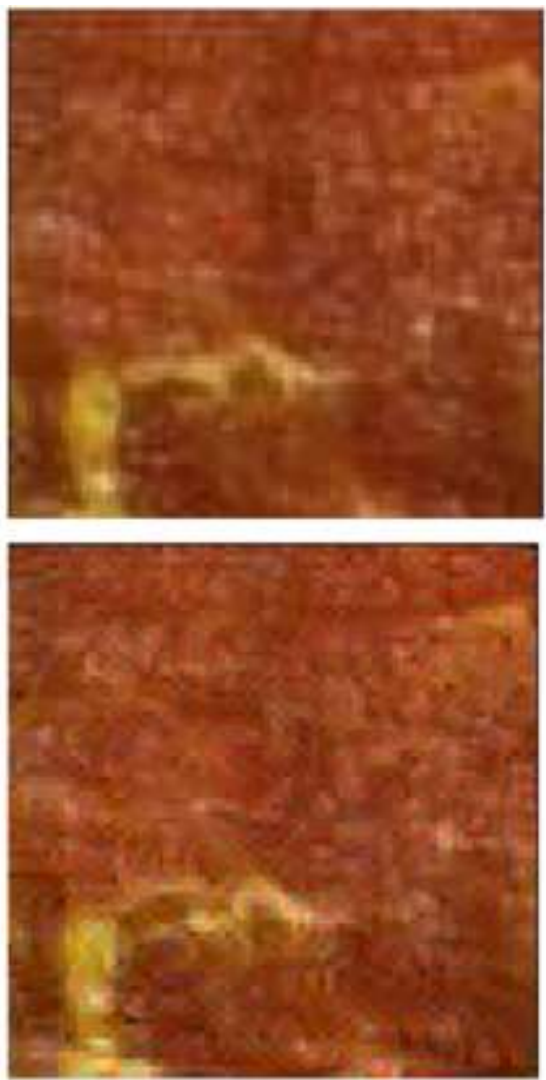

(4)

Figure 6. Illustration of the application of a filter to a data cube data during the convolution step to

The application of HSI for assessment of chemical, textural and structural characteristics of meat was

produce a feature map 1-b. (2) Image with three channels resulting from a hyperspectral data obtained from a meat samples. The three channels for this images corresponds to scores from the first three principal components applied to spectral data. (3) Example of a feature map from model by (Gatys et al.,2005) applied in data from (2), which led to fitted data as shown in (4).

\section{Conclusions} reviewed with focus on its ability to capture unique chemical and structural characteristics of meat. The assessment of chemical composition of meat allows the identification of factors associate to animal background, feeding regime, welfare i.e. unique characteristics before slaughter. But there is still an amount of evidences on its ability to handle the effect of different sources of variation on the assessment of chemical composition. The use of spatially resolved spectroscopy has been able to detect structural information related to animal background, muscle type, rigor process and ageing. Similarly the use of texture features seem to capture unique characteristics of meat.

Recently, hyperspectral systems based on snapshot imagining have become available, allowing for fast scan without the need of sample translation. This type of system promotes the development of portable devices for scanning samples with multiple illumination designs, allowing acquisition of data describing 
the absorption, scattering and texture features. The use of these abilities for the development of portable system to acquire data including absorption, scattering and texture features requires further research with the potential to provide more comprehensive description of meat. Thus further investigations should focus acquisition of this type of data (absorption, scattering and texture) to describe different levels of interaction of meat structure and its effect quality. Furthermore, data obtained with multiple illumination designs will describe much more complex interactions requiring new approaches to model hyperspectral data, such as convolutional neural networks.

\section{Acknowledgements}

The research presented on IMF was part of a Beef + Lamb New Zealand Genetics project funded by the Ministry for Business, Innovation and Employment and Beef + Lamb Zealand. The research presented in the spatially resolved experiment for bull and steers was supported by AgResearch Strategic Science Investment Fund; REPLAY (Project ID: 318920, FP7-PEOPLE-2012); Institute for the Promotion of Innovation through Science and Technology in Flanders (IWT-Flanders, grant 131777); Research Foundation Flanders (FWO); The on texture received support from Auckland University of Technology and AgResearch Strategic Science Investment Fund. We also wish to thank Dr Mustafa Farouk and Dr Steve Ranford from AgResearch for their comments and suggestions on this manuscript.

\section{References}

Achanta, R., Shaji, A., Smith, K., Lucchi, A., Fua, P., \& Süsstrunk, S. (2012). SLIC superpixels compared to state-of-the-art superpixel methods. [Article]. IEEE Transactions on Pattern Analysis and Machine Intelligence, 34(11), 2274-2281. doi: 10.1109/TPAMI.2012.120

Aernouts, B., Do Trong, N. N., Watté, R., Bruggeman, W., Tsuta, M., Verboven, P., Nicolaï, B., \& Saeys, W. (2011). Food quality control by combining light propagation models with multiple vis/NIR reflectance measurements. NIR news, 22, 14-16.

Al-Sarayreh, M., Reis, M., Yan, W. Y., \& Klette, R. (2017). Detection of Adulteration in Red Meat Species Using Hyperspectral Imaging,. Paper presented at the Pacific-Rim Symposium on Image and Video Technology (PSIVT), Wuhan, China.

Al-Sarayreh, M., Reis, M. M., Yan, Q. W., \& Klette, R. (2018). Detection of Red-Meat Adulteration by Deep Spectral-Spatial Features in Hyperspectral Images. Journal of Imaging, 4(63).

Ambroise, C., \& McLachlan, G. J. (2002). Selection bias in gene extraction on the basis of microarray gene-expression data. [Article]. Proceedings of the National Academy of Sciences of the United States of America, 99(10), 6562-6566. doi: 10.1073/pnas.102102699

Amigo, J. M., Babamoradi, H., \& Elcoroaristizabal, S. (2015). Hyperspectral image analysis. A tutorial. [Review]. Analytica Chimica Acta, 896, 34-51. doi: 10.1016/j.aca.2015.09.030

Astruc, T. (2014). CARCASS COMPOSITION, MUSCLE STRUCTURE, AND CONTRACTION Encyclopedia of Meat Sciences (Second Edition) (pp. 148-166). Oxford: Academic Press.

Barnes, R. J., Dhanoa, M. S., \& Lister, S. J. (1989). Standard normal variate transformation and detrending of near-infrared diffuse reflectance spectra. [Article]. Applied Spectroscopy, 43(5), 772777. doi: $10.1366 / 0003702894202201$

Bengio, Y., Courville, A., \& Vincent, P. (2013). Representation learning: A review and new perspectives. [Article]. IEEE Transactions on Pattern Analysis and Machine Intelligence, 35(8), 1798-1828. doi: 10.1109/TPAMI.2013.50

Borin, A., Ferrão, M. F., Mello, C., Maretto, D. A., \& Poppi, R. J. (2006). Least-squares support vector machines and near infrared spectroscopy for quantification of common adulterants in powdered milk. [Article]. Analytica Chimica Acta, 579(1), 25-32. doi: 10.1016/j.aca.2006.07.008

Chen, Q., Zhao, J., Fang, C. H., \& Wang, D. (2007). Feasibility study on identification of green, black and Oolong teas using near-infrared reflectance spectroscopy based on support vector machine 
(SVM). [Article]. Spectrochimica Acta - Part A: Molecular and Biomolecular Spectroscopy, 66(3), 568-574. doi: 10.1016/j.saa.2006.03.038

Chen, T., Morris, J., \& Martin, E. (2007). Gaussian process regression for multivariate spectroscopic calibration. [Article]. Chemometrics and Intelligent Laboratory Systems, 87(1), 85-97. doi: 10.1016/j.chemolab.2006.09.004

Chen, Y., Jiang, H., Li, C., Jia, X., \& Ghamisi, P. (2016). Deep Feature Extraction and Classification of Hyperspectral Images Based on Convolutional Neural Networks. [Article]. IEEE Transactions on Geoscience and Remote Sensing, 54(10), 6232-6251. doi: 10.1109/TGRS.2016.2584107

Chen, Y., Lin, Z., Zhao, X., Wang, G., \& Gu, Y. (2014). Deep learning-based classification of hyperspectral data. [Article]. IEEE Journal of Selected Topics in Applied Earth Observations and Remote Sensing, 7(6), 2094-2107. doi: 10.1109/JSTARS.2014.2329330

Chen, Y. N., Sun, D. W., Cheng, J. H., \& Gao, W. H. (2016). Recent Advances for Rapid Identification of Chemical Information of Muscle Foods by Hyperspectral Imaging Analysis. [Review]. Food Engineering Reviews, 8(3), 336-350. doi: 10.1007/s12393-016-9139-1

Cheng, J. H., Nicolai, B., \& Sun, D. W. (2017). Hyperspectral imaging with multivariate analysis for technological parameters prediction and classification of muscle foods: A review. [Review]. Meat Science, 123, 182-191. doi: 10.1016/j.meatsci.2016.09.017

Cheng, W., Sun, D.-W., Pu, H., \& Liu, Y. (2016). Integration of spectral and textural data for enhancing hyperspectral prediction of $\mathrm{K}$ value in pork meat. LWT - Food Science and Technology, 72, 322329. doi: https://doi.org/10.1016/..Iwt.2016.05.003

Chong, I. G., \& Jun, C. H. (2005). Performance of some variable selection methods when multicollinearity is present. [Article]. Chemometrics and Intelligent Laboratory Systems, 78(1), 103-112. doi: 10.1016/j.chemolab.2004.12.011

Cluff, K., Naganathan, G. K., Subbiah, J., Lu, R., Calkins, C. R., \& Samal, A. (2008). Optical scattering in beef steak to predict tenderness using hyperspectral imaging in the VIS-NIR region. Sensing and Instrumentation for Food Quality and Safety, 2(3), 189-196.

Cluff, K., Naganathan, G. K., Subbiah, J., Samal, A., \& Calkins, C. R. (2013). Optical scattering with hyperspectral imaging to classify longissimus dorsi muscle based on beef tenderness using multivariate modeling. Meat Science, 95(1), 42-50. doi: https://doi.org/10.1016/i.meatsci.2013.04.014

Craigie, C. R., Johnson, P. L., Shorten, P. R., Charteris, A., Maclennan, G., Tate, M. L., Agnew, M. P., Taukiri, K. R., Stuart, A. D., \& Reis, M. M. (2017). Application of Hyperspectral imaging to predict the $\mathrm{pH}$, intramuscular fatty acid content and composition of lamb $\mathrm{M}$. longissimus lumborum at 24 h post mortem. [Article]. Meat Science, 132, 19-28. doi: 10.1016/j.meatsci.2017.04.010

Dahm, D., \& Dahm, K. (2001). Near-Infrared Technologies in the Agricultural and Food Industries. St. Paul, MN: American Association of Cereal Chemists.

Dahm, K. D., \& Dahm, D. J. (2013). Separating the effects of scatter and absorption using the representative layer. [Article]. Journal of Near Infrared Spectroscopy, 21(5), 351-357. doi: $10.1255 /$ jnirs. 1062

Di Cataldo, S., \& Ficarra, E. (2017). Mining textural knowledge in biological images: Applications, methods and trends. [Review]. Computational and Structural Biotechnology Journal, 15, 56-67. doi: 10.1016/j.csbj.2016.11.002

Elmasry, G., Sun, D. W., \& Allen, P. (2012). Near-infrared hyperspectral imaging for predicting colour, pH and tenderness of fresh beef. Journal of Food Engineering, 110(1), 127-140.

Fei-Fei, L., Johnson, J., \& Yeung, S. (2017). Convolutional Neural Networks Retrieved 29/01/2017, from http://cs231n.stanford.edu/slides/2017/cs231n 2017 lecture5.pdf

Feng, C. H., Makino, Y., Oshita, S., \& García Martín, J. F. (2018). Hyperspectral imaging and multispectral imaging as the novel techniques for detecting defects in raw and processed meat products: Current state-of-the-art research advances. [Review]. Food Control, 84, 165-176. doi: 10.1016/j.foodcont.2017.07.013

Feng, Y. Z., \& Sun, D. W. (2013). Near-infrared hyperspectral imaging in tandem with partial least squares regression and genetic algorithm for non-destructive determination and visualization of Pseudomonas loads in chicken fillets. [Article]. Talanta, 109, 74-83. doi: 10.1016/j.talanta.2013.01.057

Garrido-Novell, C., Garrido-Varo, A., Pérez-Marín, D., \& Guerrero, J. E. (2018). Using spectral and textural data extracted from hyperspectral near infrared spectroscopy imaging to discriminate between processed pork, poultry and fish proteins. Chemometrics and Intelligent Laboratory Systems, 172, 90-99. doi: https://doi.org/10.1016/i.chemolab.2017.11.011

Gatys, L. A., Ecker, A. S., \& Bethge, M. (2015). Texture synthesis using convolutional neural networks. Paper presented at the Advances in Neural Information Processing Systems. 
Geelen, B., Jayapala, M., Tack, N., \& Lambrechts, A. (2013). Low complexity image processing for a high throughput, low latency snapshot multispectral imager with integrated, tiled filters. Paper presented at the Proceedings of SPIE - The International Society for Optical Engineering.

Geladi, P. (2003). Chemometrics in spectroscopy. Part 1. Classical chemometrics. [Review]. Spectrochimica Acta - Part B Atomic Spectroscopy, 58(5), 767-782. doi: 10.1016/S05848547(03)00037-5

Gibson, N. P., Aigrain, S., Roberts, S., Evans, T. M., Osborne, M., \& Pont, F. (2012). A Gaussian process framework for modelling instrumental systematics: Application to transmission spectroscopy. [Article]. Monthly Notices of the Royal Astronomical Society, 419(3), 2683-2694. doi: 10.1111/j.1365-2966.2011.19915.x

Guo, T., Huang, M., Zhu, Q., Guo, Y., \& Qin, J. (2018). Hyperspectral image-based multi-feature integration for TVB-N measurement in pork. Journal of Food Engineering, 218, 61-68. doi: https://doi.org/10.1016/i.jfoodeng.2017.09.003

Haralick, R. M. (1979). STATISTICAL AND STRUCTURAL APPROACHES TO TEXTURE. 45-46.

Hastie, T., Tibshirani, R., \& Friedman, J. (2008). The Elements of Statistical Learning (2nd edition ed.). New York: Springer.

Hu, W., Huang, Y., Wei, L., Zhang, F., \& Li, H. (2015). Deep convolutional neural networks for hyperspectral image classification. [Article]. Journal of Sensors, 2015. doi: 10.1155/2015/258619

Huff-Lonergan, E., \& Lonergan, S. M. (2005). Mechanisms of water-holding capacity of meat: The role of postmortem biochemical and structural changes. Meat Science, 71(1), 194-204.

Jacques, S. L. (2013). Optical properties of biological tissues: A review. [Review]. Physics in Medicine and Biology, 58(11), R37-R61. doi: 10.1088/0031-9155/58/11/R37

Juan, A. d., Tauler, R., Dyson, R., Marcolli, C., Rault, M., \& Maeder, M. (2004). Spectroscopic imaging and chemometrics: a powerful combination for global and local sample analysis. TrAC Trends in Analytical Chemistry, 23(1), 70-79. doi: https://doi.org/10.1016/S0165-9936(04)00101-3

Kamruzzaman, M., ElMasry, G., Sun, D.-W., \& Allen, P. (2012). Non-destructive prediction and visualization of chemical composition in lamb meat using NIR hyperspectral imaging and multivariate regression. Innovative Food Science \& Emerging Technologies, 16, 218-226. doi: https://doi.org/10.1016/j.ifset.2012.06.003

Kamruzzaman, M., ElMasry, G., Sun, D.-W., \& Allen, P. (2013). Non-destructive assessment of instrumental and sensory tenderness of lamb meat using NIR hyperspectral imaging. Food Chemistry, 141(1), 389-396. doi: http://dx.doi.org/10.1016/j.foodchem.2013.02.094

Kamruzzaman, M., Makino, Y., \& Oshita, S. (2015). Non-invasive analytical technology for the detection of contamination, adulteration, and authenticity of meat, poultry, and fish: A review. [Review]. Analytica Chimica Acta, 853(1), 19-29. doi: 10.1016/j.aca.2014.08.043

Keresztes, J. C., Goodarzi, M., \& Saeys, W. (2016). Real-time pixel based early apple bruise detection using short wave infrared hyperspectral imaging in combination with calibration and glare correction techniques. [Article]. Food Control, 66, 215-226. doi: 10.1016/j.foodcont.2016.02.007

Klette, R. (2014). Concise Computer Vision. London: Springer.

Lecun, Y., Bengio, Y., \& Hinton, G. (2015). Deep learning. [Review]. Nature, 521(7553), 436-444. doi: $10.1038 /$ nature 14539

Li, B., Morris, J., \& Martin, E. B. (2002). Model selection for partial least squares regression. [Article]. Chemometrics and Intelligent Laboratory Systems, 64(1), 79-89. doi: 10.1016/S01697439(02)00051-5

Li, H., Liang, Y., Xu, Q., \& Cao, D. (2009). Key wavelengths screening using competitive adaptive reweighted sampling method for multivariate calibration. [Article]. Analytica Chimica Acta, 648(1), 77-84. doi: 10.1016/j.aca.2009.06.046

Li, Y., Zhang, H., \& Shen, Q. (2017). Spectral-spatial classification of hyperspectral imagery with 3D convolutional neural network. [Article]. Remote Sensing, 9(1). doi: 10.3390/rs9010067

Liu, Y., Pu, H., \& Sun, D. W. (2017). Hyperspectral imaging technique for evaluating food quality and safety during various processes: A review of recent applications. [Review]. Trends in Food Science and Technology, 69, 25-35. doi: 10.1016/j.tifs.2017.08.013

Ma, J., Pu, H., Sun, D. W., Gao, W., Qu, J. H., \& Ma, K. Y. (2015). Application of Vis-NIR hyperspectral imaging in classification between fresh and frozen-thawed pork Longissimus Dorsi muscles. [Article]. International Journal of Refrigeration, 50, 10-18. doi: 10.1016/j.jirefrig.2014.10.024

Makantasis, K., Karantzalos, K., Doulamis, A., \& Doulamis, N. (2015). Deep supervised learning for hyperspectral data classification through convolutional neural networks. Paper presented at the International Geoscience and Remote Sensing Symposium (IGARSS).

Martens, H., \& Naes, T. (1992). Multivariate calibration. Chichester John Wiley \& Sons. 
Mollazade, K., Omid, M., Tab, F. A., \& Mohtasebi, S. S. (2012). Principles and Applications of Light Backscattering Imaging in Quality Evaluation of Agro-food Products: A Review. [Review]. Food and Bioprocess Technology, 5(5), 1465-1485. doi: 10.1007/s11947-012-0821-X

Naganathan, G. K., Cluff, K., Samal, A., Calkins, C. R., Jones, D. D., Lorenzen, C. L., \& Subbiah, J. (2015). Hyperspectral imaging of ribeye muscle on hanging beef carcasses for tenderness assessment. [Article]. Computers and Electronics in Agriculture, 116, 55-64. doi: 10.1016/j.compag.2015.06.006

Ogata, T., \& Yamasaki, Y. (1997). Ultra-high-resolution scanning electron microscopy of mitochondria and sarcoplasmic reticulum arrangement in human red, white, and intermediate muscle fibers. [Article]. Anatomical Record, 248(2), 214-223. doi: 10.1002/(SICl)10970185(199706)248:2<214::AID-AR8>3.0.CO;2-S

Peng, Y., Wu, J., \& Chen, J. (2009). Prediction of beef quality attributes using hyperspectral scattering imaging technique. Paper presented at the American Society of Agricultural and Biological Engineers Annual International Meeting 2009, ASABE 2009.

$\mathrm{Pu}, \mathrm{H}$., Sun, D.-W., Ma, J., \& Cheng, J.-H. (2015). Classification of fresh and frozen-thawed pork muscles using visible and near infrared hyperspectral imaging and textural analysis. Meat Science, 99, 81-88. doi: https://doi.org/10.1016/i.meatsci.2014.09.001

Pu, H., Sun, D.-W., Ma, J., Liu, D., \& Kamruzzaman, M. (2014). Hierarchical variable selection for predicting chemical constituents in lamb meats using hyperspectral imaging. Journal of Food Engineering, 143, 44-52. doi: https://doi.org/10.1016/j.jfoodeng.2014.06.025

Pu, H., Sun, D. W., Ma, J., Liu, D., \& Cheng, J. H. (2014). Using Wavelet Textural Features of Visible and Near Infrared Hyperspectral Image to Differentiate Between Fresh and Frozen-Thawed Pork. [Article]. Food and Bioprocess Technology, 7(11), 3088-3099. doi: 10.1007/s11947-014-1330-x

Ranasinghesagara, J., Nath, T. M., Wells, S. J., Weaver, A. D., Gerrard, D. E., \& Yao, G. (2010). Imaging optical diffuse reflectance in beef muscles for tenderness prediction. [Article]. Meat Science, 84(3), 413-421. doi: 10.1016/j.meatsci.2009.09.010

Ranasinghesagara, J., \& Yao, G. (2007). Imaging 2D optical diffuse reflectance in skeletal muscle. Optics Express, 15, 3998-4007.

Rinnan, A., Berg, F. v. d., \& Engelsen, S. B. (2009a). Review of the most common pre-processing techniques for near-infrared spectra. [Review]. TrAC - Trends in Analytical Chemistry, 28(10), 1201-1222. doi: 10.1016/j.trac.2009.07.007

Rinnan, A.., Berg, F. v. d., \& Engelsen, S. B. (2009b). Review of the most common pre-processing techniques for near-infrared spectra. TrAC Trends in Analytical Chemistry, 28(10), 1201-1222. doi: https://doi.org/10.1016/j.trac.2009.07.007

Saeys, W., Trong, N. N. D., Watté, R., Tsuta, M., Ramon, H., \& Nicolaï, B. M. (2010). Optical characterization of biological material: A multiscale approach. Paper presented at the American Society of Agricultural and Biological Engineers Annual International Meeting 2010, ASABE 2010.

Savitzky, A., \& Golay, M. J. E. (1964). Smoothing and Differentiation of Data by Simplified Least Squares Procedures. [Article]. Analytical Chemistry, 36(8), 1627-1639. doi: 10.1021/ac60214a047

Schmidhuber, J. (2015). Deep Learning in neural networks: An overview. [Review]. Neural Networks, 61, 85-117. doi: 10.1016/j.neunet.2014.09.003

Shorten, P. R., \& Sneyd, J. (2009). A Mathematical Analysis of Obstructed Diffusion within Skeletal Muscle. Biophysical Journal, 96(12), 4764-4778. doi: https://doi.org/10.1016/j.bpj.2009.02.060

Siche, R., Vejarano, R., Aredo, V., Velasquez, L., Saldaña, E., \& Quevedo, R. (2016). Evaluation of Food Quality and Safety with Hyperspectral Imaging (HSI). [Review]. Food Engineering Reviews, 8(3), 306-322. doi: 10.1007/s12393-015-9137-8

Sjöblom, J., Svensson, O., Josefson, M., Kullberg, H., \& Wold, S. (1998). An evaluation of orthogonal signal correction applied to calibration transfer of near infrared spectra. [Conference Paper]. Chemometrics and Intelligent Laboratory Systems, 44(1-2), 229-244. doi: 10.1016/S01697439(98)00112-9

Svetnik, V., Liaw, A., Tong, C., \& Wang, T. (2004) Application of Breiman's Random Forest to modeling structure-activity relationships of pharmaceutical molecules. Vol. 3077. Lecture Notes in Computer Science (including subseries Lecture Notes in Artificial Intelligence and Lecture Notes in Bioinformatics) (pp. 334-343).

Tornberg, E., Wahlgren, M., Brøndum, J., \& Engelsen, S. B. (2000). Pre-rigor conditions in beef under varying temperature- and $\mathrm{pH}$-falls studied with rigometer, NMR and NIR. Food Chemistry, 69(4), 407-418. 
Van Beers, R., Aernouts, B., Deserranno, D., Watté, R., \& Saeys, W. (2017). A Handheld Multispectral Sensor for the Separation of Scattering and Absorption Properties. Paper presented at the The 18th International Conference on Near Infrared Spectroscopy, Copenhagen, Denmark.

Van Beers, R., Aernouts, B., Reis, M. M., \& Saeys, W. (2017). Anisotropic light propagation in bovine muscle tissue depends on the initial fiber orientation, muscle type and wavelength. [Article]. Optics Express, 25(18), 22082-22095. doi: 10.1364/OE.25.022082

Van Beers, R., Kokawa, M., Aernouts, B., Watté, R., De Smet, S., \& Saeys, W. (2018). Evolution of the bulk optical properties of bovine muscles during wet aging. Meat Science, 136, 50-58. doi: https://doi.org/10.1016/i.meatsci.2017.10.010

Verrelst, J., Alonso, L., Rivera Caicedo, J. P., Moreno, J., \& Camps-Valls, G. (2013). Gaussian process retrieval of chlorophyll content from imaging spectroscopy data. [Article]. IEEE Journal of Selected Topics in Applied Earth Observations and Remote Sensing, 6(2), 867-874. doi: 10.1109/JSTARS.2012.2222356

Wallis, T. S. A., Funke, C. M., Ecker, A. S., Gatys, L. A., Wichmann, F. A., \& Bethge, M. (2017). A parametric texture model based on deep convolutional features closely matches texture appearance for humans. [Article]. Journal of Vision, 17(12). doi: 10.1167/17.12.5

Wu, J., Peng, Y., Li, Y., Wang, W., Chen, J., \& Dhakal, S. (2012). Prediction of beef quality attributes using VIS/NIR hyperspectral scattering imaging technique. Journal of Food Engineering, 109(2), 267-273.

Wu, J. H., Peng, Y. K., Chen, J. J., Wang, W., Gao, X. D., \& Huang, H. (2010). Study of spatially resolved hyperspectral scattering images for assessing beef quality characteristics. Guang Pu Xue Yu Guang Pu Fen Xi/Spectroscopy and Spectral Analysis, 30(7), 1815-1819.

Xia, J., Weaver, A., Gerrard, D. E., \& Yao, G. (2006). Monitoring sarcomere structure changes in whole muscle using diffuse light reflectance. [Article]. Journal of Biomedical Optics, 11(4). doi: $10.1117 / 1.2234278$

Xia, J. J., Berg, E. P., Lee, J. W., \& Yao, G. (2007). Characterizing beef muscles with optical scattering and absorption coefficients in VIS-NIR region. [Article]. Meat Science, 75(1), 78-83. doi: 10.1016/j.meatsci.2006.07.002

Xiong, Z., Sun, D. W., Pu, H., Zhu, Z., \& Luo, M. (2015). Combination of spectra and texture data of hyperspectral imaging for differentiating between free-range and broiler chicken meats. [Article]. LWT - Food Science and Technology, 60(2), 649-655. doi: 10.1016/j.Iwt.2014.10.021

Xiong, Z., Sun, D. W., Zeng, X. A., \& Xie, A. (2014). Recent developments of hyperspectral imaging systems and their applications in detecting quality attributes of red meats: A review. Journal of Food Engineering, 132, 1-13.

Zayed, N., \& Elnemr, H. A. (2015). Statistical Analysis of Haralick Texture Features to Discriminate Lung Abnormalities. [Article]. International Journal of Biomedical Imaging, 2015. doi: $10.1155 / 2015 / 267807$

Zhang, Y., Cong, Q., Xie, Y., JingxiuYang, \& Zhao, B. (2008). Quantitative analysis of routine chemical constituents in tobacco by near-infrared spectroscopy and support vector machine. [Article]. Spectrochimica Acta - Part A: Molecular and Biomolecular Spectroscopy, 71(4), 1408-1413. doi: 10.1016/j.saa.2008.04.020 